\title{
Double Dependent Locality
}

National Cancer Institute

\section{Source}

National Cancer Institute. Double Dependent Locality. NCI Thesaurus. Code C48951.

A locality or minor thoroughfare the address for which must be specified in relationship to a more major one. 